Original Paper http://ajol.info/index.php/ijbcs http://indexmedicus.afro.who.int

\title{
Diversité floristique et structure de la végétation ligneuse des parcs arborés de la zone soudanienne du Tchad
}

\author{
Hervé MBAIYETOM ${ }^{1,2^{*}}$, Marie Louise AVANA TIENTCHEU ${ }^{2}$, \\ Martin TCHAMBA NGANKAM ${ }^{2}$ et Junior Baudoin WOUOKOUE TAFFO ${ }^{3}$ \\ ${ }^{1}$ Département des Sciences Biologiques, Faculté des Sciences et Techniques, Université de Doba, BP 03 Doba, \\ Tchad. \\ ${ }^{2}$ Département de Foresterie, Faculté d'Agronomie et des Sciences Agricoles, Université de Dschang, BP 222 \\ Dschang, Cameroun. \\ ${ }^{3}$ Département des Sciences Biologiques, Faculté des Sciences, Université de Maroua, BP 814 Maroua, \\ Cameroun. \\ *Auteur correspondant ; E-mail : hmbaiyetom@gmail.com ; Tél : (+235) 68759541/95733773
}

Received: 12-10-2020 Accepted: 29-01-2021 $\quad$ Published: 28-02-2021

\section{RESUME}

Les parcs arborés du Tchad subissent une dégradation marquée par une diminution importante du couvert végétal et des ressources ligneuses en raison de la péjoration climatique et de l'anthropisation. L'objectif de cette étude était d'évaluer la diversité floristique des parcs arborés du Département de la Nya (Sud du Tchad) et de caractériser leurs paramètres écologiques. Les données floristiques ont été collectées sur 174 relevés de $2500 \mathrm{~m}^{2}$ chacun. Au total, 29 espèces ligneuses réparties en 29 genres et 18 familles ont été recensées. La densité moyenne est de 28,528 tiges/ha et la surface terrière moyenne est de 4,077 $\mathrm{m}^{2} / \mathrm{ha}$. L'indice de diversité de Shannon était de 3,043 et l'équitabilité de Piélou était de 0,626. Les familles les plus dominantes sont les Fabaceae, Sapotaceae et Anacardiaceae. La structure en diamètre a mis en évidence une prédominance d'individus adultes par rapport aux individus de petit diamètre (4,60\%). La chorologie de la flore est caractérisée par la dominance des espèces soudano-zambésiennes (37,93\%). Le spectre de formes biologiques montre une prédominance des mésophanérophytes $(65,51 \%)$ et des microphanérophytes $(31,03 \%)$. Ces résultats peuvent servir de référence dans le suivi de la dynamique de la végétation des parcs arborés soumis aux variations climatiques et aux pressions anthropiques.

(C) 2020 International Formulae Group. All rights reserved.

Mots clés : Caractéristique floristique, parcs arborés, types biologiques, types phytogéographiques, zone soudanienne, Tchad.

\section{Floristic diversity and vegetation structure of the woody species of the parklands in the sudano-savanna zone of Chad}

\begin{abstract}
Woodlands vegetation in Chad have been severely degraded due to climatic deterioration and high anthropogenic pressure which occurred in recent decades, causing significant reduction in plant cover and woody resources. The aim of this study was to evaluate the floristic diversity of the woody species in sudanian parklands
\end{abstract}


in the Nya Division (Southern Chad) and to characterize their ecological parameters. The floristic data were collected on 174 floristic surveys' plots of $2500 \mathrm{~m}^{2}$ each. A total of 29 woody species belonging to 29 genera and 18 families were recorded. The average density was $28,528 \mathrm{stems} /$ ha and the average basal area for standing stems was of $4,077 \mathrm{~m}^{2} / \mathrm{ha}$. The Shannon diversity index was 3,043 and Pielou evenness index was 0,626 . The most dominant families were Fabaceae, Sapotaceae and Anacardiaceae. Diameter structures showed a predominance of adult trees over small-diameter stems $(4.60 \%)$. The chorology of the flora is characterized by the predominance of Sudano-Zambesian species (37.93\%). The spectrum of detailed biological forms for the phanerophyte' subtypes shows a predominance of mesophanerophytes $(65.51 \%)$ and microphanerophytes (31.03\%). These results can be used as a baseline for monitoring the dynamics of the vegetation in savanna woodland areas subject to climatic and anthropogenic pressures.

(C) 2020 International Formulae Group. All rights reserved.

Keywords: Floristic characteristics, woodlands, biological spectrum, phytogeographic types, sudano-savanna zone, Chad.

\section{INTRODUCTION}

Les espèces ligneuses des parcs arborés en Afrique au Sud du Sahara sont très importantes pour les populations humaines en raison de leur contribution à la satisfaction des besoins en alimentation, santé, énergie, revenus et autres nécessités du bien-être humain (Akinnifesi et al., 2008 ; Oumorou et al., 2010 ; Manzo et al., 2017). Elles procurent de nombreux services et produits forestiers ligneux et non ligneux d'origine végétale parmi lesquels les feuilles, fleurs, fruits, graines, écorces, sèves, fibres, rhizomes, etc. Ces ligneux et leurs habitats subissent des perturbations liées aux actions anthropiques et aux changements climatiques, qui menacent leur survie alors même que leurs caractéristiques écologiques, morphologiques, génétiques et leurs usages restent très peu documentés (Manzo et al., 2017). Par conséquent, la dégradation de ces ressources ligneuses affecte tous les domaines agro et socioécologiques de la vie aussi bien au niveau local que global.

Au Tchad, la situation des parcs arborés se caractérise par une dégradation accélérée des sols déjà fragilisés par un nombre élevé d'aléas climatiques, puis la poussée démographique avec son corollaire de demande croissante en terre cultivable et de bois d'énergie (Djimadoum, 2015; Madjigoto et al., 2015). La combinaison de facteurs influe certainement sur la composition des espèces ligneuses et la structure de la végétation qui en résulte. Malgré l'importance écologique et socioéconomique de ces parcs arborés qui sont gérés traditionnellement depuis de nombreuses générations, peu d'informations existe sur leur diversité floristique et leurs paramètres écologiques. Les parcs arborés du Département de la Nya ne font pas exception à ce gap de connaissance.

La caractérisation des parcs arborés et des communautés d'espèces qui leurs sont inféodées est fondamentale pour évaluer leur importance dans le maintien et la pérennisation de la biodiversité. En effet, selon Kent et Coker (2003), les données sur la végétation sont d'une grande utilité dans la résolution des problèmes écologiques, notamment comme une base de prédiction des futurs changements possibles. Cette opinion est soutenue par Mbaygone (2008) pour qui les caractéristiques floristiques globales ainsi que l'organisation des phytocénoses sont des éléments indicateurs de stabilité ou de dégradation d'un milieu pouvant servir comme instrument d'alerte ou de prise de décision pour les gestionnaires des écosystèmes. Ainsi, Wouokoue (2018) précise que la protection des ressources contre les différents facteurs de dégradation n'est possible que si l'on dispose d'une bonne connaissance sur la diversité floristique et sur les traits fonctionnels des écosystèmes qu'elle reflète. La gestion durable de ces ressources forestières passe nécessairement par une meilleure connaissance de leur diversité qui permettra de mieux comprendre ses éléments constitutifs et d'effectuer un suivi de sa dynamique au cours du temps (Larwanou et 
Saadou, 2005). La présente étude a pour objectif d'évaluer la diversité floristique et de caractériser les paramètres écologiques des parcs arborés du Département de la Nya au Sud du Tchad.

\section{MATERIEL ET METHODES Site d'étude}

Situé au centre-est de la province du Logone Oriental, dans l'extrême sud du Tchad, entre $8^{\circ}$ et $9^{\circ} 15^{\prime}$ de latitude Nord et entre $16^{\circ} 20^{\prime}$ et $16^{\circ} 50^{\prime}$ de longitude Est, le Département de la Nya occupe une superficie de $2954 \mathrm{~km}^{2}$ et représente $22,7 \%$ de la province et $0,23 \%$ du territoire national (Figure 1). La densité moyenne de la population est de 47 habitants $/ \mathrm{km}^{2}$ alors que la moyenne nationale est de 8,6 habitants $/ \mathrm{km}^{2}$ (INSEED, 2009). Cette densité résulterait d'un niveau de fécondité très élevé au Tchad en général ainsi qu'à la sédentarisation de nombreux migrants (Magrin et al., 2011).

Le Département de la Nya est sous l'influence du climat tropical de type soudanien caractérisé par une courte saison sèche (novembre-mars) et une longue saison pluvieuse (avril-octobre). Les précipitations moyennes annuelles sont de $1000 \mathrm{~mm}$ d'eau. Cependant, au cours de ces dernières décennies, elles connaissent une forte variabilité interannuelle. La température varie selon les différentes saisons de l'année avec des maxima se situant autour de $40{ }^{\circ} \mathrm{C}$ pour les mois de mars et avril et les minima autour de $20{ }^{\circ} \mathrm{C}$ en décembre et janvier. Le réseau hydrographique est constitué du fleuve Logone avec ses affluents : Pendé, Nya, Lim, Loule et Ouham-Pendé. Les sols sont ferrugineux, ferralitiques ou hydromorphes. La végétation est constituée des arbres isolés dans les parcelles agricoles ou parcs arborés, des galeries forestières, des savanes arbustives et des réliques des savanes arborées. Les espèces les plus dominantes de ces formations sont Parkia biglobosa, Vitellaria paradoxa, Pterocarpus lucens, Kaya senegalensis, Daniellia oliveri, Anogeissus leiocarpa, Burkea africana, Lophira lanceolata, Prosopis africana... (Djeralar, 2010). La population pratique l'agriculture et l'élevage comme leurs principales activités économiques.

\section{Méthodologie \\ Relevés de la végétation}

Les relevés floristiques ont été effectués sur 24 sites répartis dans les six cantons que compte le Département de Nya au Sud du Tchad. Au niveau de chaque site, l'inventaire floristique s'est fait dans les placettes carrés de $50 \mathrm{~m}$ de côté $\left(2500 \mathrm{~m}^{2}\right)$, équidistantes de 200 $\mathrm{m}$ en moyenne suivant un échantillonnage systématique tel que décrit par Glélé et al. (2016) et Thiombiano et al. (2016) (Figure 2). Au total, 174 placettes ont été installées sur l'ensemble de la zone d'étude. Sur chaque placette, les coordonnées géographiques étaient prises à l'aide d'un GPS (Geographic Positioning System); tous les ligneux rencontrés étaient identifiés, comptabilisés et leur circonférence mesurée à 1,30 m au-dessus du sol. L'identification des espèces s'est faite sur le terrain à l'aide du lexique de noms vernaculaires des plantes au Tchad (Gaston et al., 1971) et du manuel "arbres, arbustes et lianes des zones sèches d'Afrique de l'Ouest'" (Arbonnier, 2000).

\section{Analyse des données \\ Diversité spécifique des parcs arborés}

La diversité spécifique est définie par le nombre total d'espèces et d'individus (abondance) dans un groupement donné. Les principaux indices de diversité calculés sont présentés ci-dessous.

L'indice de diversité de Shannon, H' = $-\Sigma$ pi $\times \log _{2}$ pi avec $\mathrm{H}^{\prime}=$ indice de diversité de Shannon, qui s'exprime en bit. Il varie généralement de 1 à 5 bits. Si H' $€[0 ; 2,5]$ alors $H^{\prime}$ peut être supposé faible (cas des stations spécialisées où l'on note généralement des phénomènes de dominance d'une espèce ou d'un petit nombre d'espèces sur l'ensemble des espèces de la communauté); Si H' est compris entre $[2,6 ; 3,9]$ alors il peut être supposé moyen ; Si H' est compris entre [4;5] alors il peut être supposé élevé (cas des stations isotropes où les espèces tendent vers l'équiprobabilité) $; \mathrm{pi}=\mathrm{ri} / \mathrm{r}$; où ri est le nombre d'individus de l'espèce i dans le relevé 
considéré et $\mathrm{r}$ désigne le nombre total des individus du relevé.

L'indice d'équitabilité de Piélou (E) traduit le degré de diversité atteint par rapport au maximum théorique. Il est calculé par la formule : $\mathrm{E}=\mathrm{H}^{\prime} / \mathrm{H}$ max avec $\mathrm{H} \max =\log _{2} \mathrm{~S}$ ; où $\mathrm{S}$ est le nombre total d'espèces. Il est compris entre 0 et 1 . E tend vers 0 lorsque la quasi-totalité des individus appartiennent à une seule espèce et prend la valeur 1 lorsque toutes les espèces ont exactement le même recouvrement.

La densité (D) des ligneux est calculée selon la formule $: \mathrm{D}=\mathrm{N} \times 10000 / \mathrm{S}$ Avec $\mathrm{N}$ est le nombre de ligneux et $S$ est la superficie inventoriée rapportée à l'hectare. D s'exprime en nombre de tiges/ha.

La surface terrière : Elle est notée Gi et est calculée par la formule : $\mathrm{Gi}=\sum(2 \mathrm{~d})^{2} / 4 \Pi$ ou Gi en $\mathrm{m}^{2} /$ ha et $\mathrm{d}$ est le diamètre exprimé en $\mathrm{m}$

\section{Caractéristiques écologiques des parcs arborés}

Les types biologiques et l'affinité phytogéographique de chaque espèce ont été illustrés à l'aide des spectres bruts. Les principaux paramètres évalués étaient: les types biologiques, les types phytogéographiques, l'Indice de Valeur d'Importance et la valeur d'Importance des Familles.

Les types biologiques (TB) utilisés sont ceux définis par Raunkiaer (1932) adaptés pour l'étude des formations végétales tropicales par divers auteurs (Mbaygone et al., 2008b; Thiombiano et al., 2012). Les types phytogéographiques (TP) utilisés sont basés sur les grandes subdivisions chorologiques établies pour l'Afrique (White, 1986).

L'Indice de Valeur d'Importance (IVI) des espèces $=$ Fréquence relative + Densité relative + dominance relative (pour l'espèce) et la Valeur d'Importance des Familles (VIF)= Diversité relative d'une famille + Densité relative d'une famille + dominance relative d'une famille.

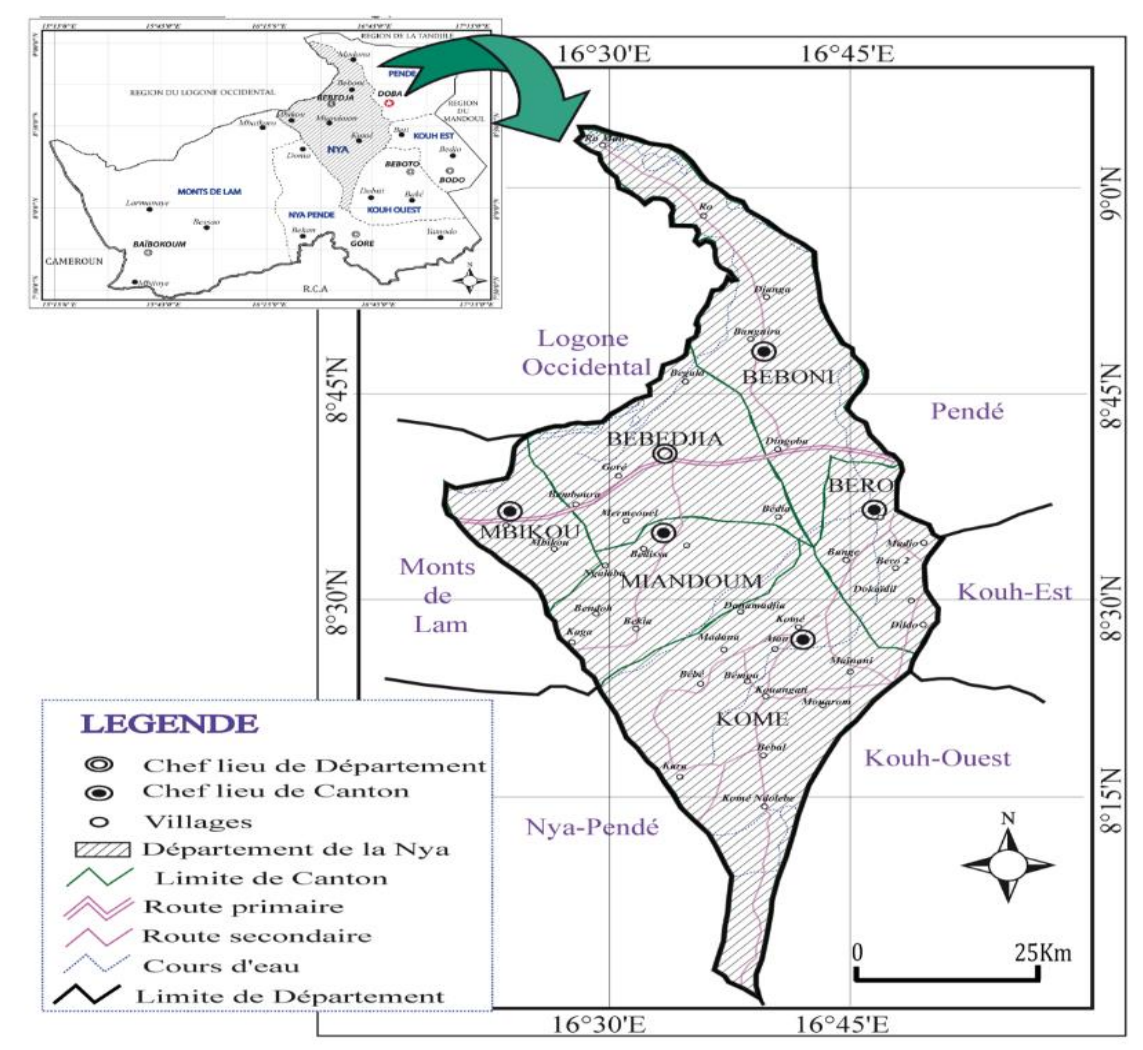

Figure 1 : Localisation des cantons d'étude dans le Département de la Nya. Source : INSEED (2010). 


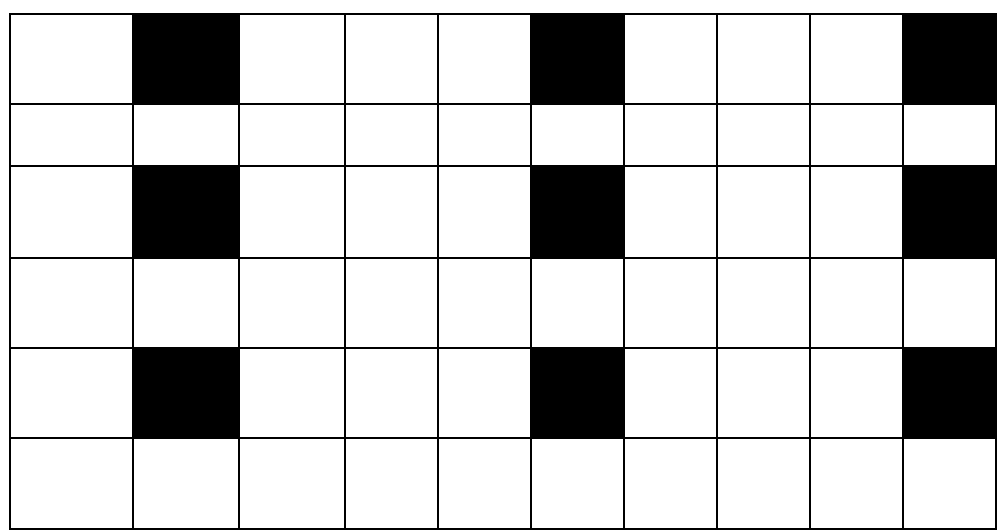

Figure 2 : Dispositif d'inventaire systématique (adapté selon Glele et al. (2016).

Les placettes en carré plein ont une superficie de $2500 \mathrm{~m}^{2}(50 \mathrm{~m}$ x 50m); Deux placettes consécutives sont équidistantes de $200 \mathrm{~m}$.

\section{RESULTATS}

Richesse floristique et diversité des parcs arborés du Département de la Nya

L'inventaire de la flore a permis de recenser au total 29 espèces végétales ligneuses, réparties en 29 genres et 18 familles (selon la classification APG III). La valeur de l'indice de diversité de Shannon est de 3,043 et celle de l'indice d'équitabilité de Piélou est de 0,626 pour l'ensemble des placettes et des cantons échantillonnés.

\section{Caractéristiques floristiques des parcs arborés}

L'espèce Vitellaria paradoxa apparaît dans 137 des 174 relevés, avec une fréquence relative de 78,73\%, suivie de Parkia biglobosa et de Daniellia oliveri apparaissant dans 105 et 67 des 174 relevés avec respectivement des fréquences relatives de $(60,34 \%)$ et $(38,50 \%)$ (Tableau 1). Quatre espèces ont eu moins de $1 \%$ de fréquence relative, il s'agit de Ficus capensis, Carica papaya, sclerocarya birrea et Strychnos spinosa.

Sur les 1241 individus recensés dans cette étude, Vitellaria paradoxa avec 435 tiges est l'espèce la plus abondante avec 35,05\% d'abondance relative, suivie de Parkia biglobosa (282 individus) soit 12,52\% d'abondance relative et de Daniellia oliveri (116 individus) soit $10,62 \%$ d'abondance relative (Tableau 1). Les neuf espèces les plus abondantes ont eu une abondance relative cumulée de $91,53 \%$. Une grande proportion d'espèces (10 espèces), soit $34,48 \%$ sont représentées par moins de 5 individus; 4 espèces sont représentées par 2 individus et 3 espèces (soit 10,34\%) sont représentées par un seul individu.

Les espèces écologiquement importantes sont Vitellaria paradoxa avec un indice de valeur d'importance (IVI) 139,42\%, suivie de Parkia biglobosa $(117,11 \%)$ et Daniellia oliveri $(58,33 \%)$ (Tableau 1). Les espèces les moins importantes ayant un IVI inférieur à $1 \%$ sont: Carica papaya, Sclerocarya birrea et Strychnos spinosa.

\section{Structure diamétrique des parcs arborés}

La classe de diamètre $[20 ; 50[\mathrm{~cm}$ avec 820 individus est la plus représentée suivie de celle de $[50 ; 100[\mathrm{~cm}$ avec 355 individus. $\mathrm{La}$ classe de diamètre $[100 ; \rightarrow[$ est la moins représentée avec 3 individus (Figure 3 ).

\section{Densité et surface terrière des espèces dans les parcs arborés}

Mille deux cent quarante un (1241) individus ont été recensés sur une superficie de 43,5 ha, ce qui représente une densité moyenne de 28,528 tiges/ha. Vitellaria paradoxa est l'espèce la plus dense avec 10 tiges/ha suivie 
de Parkia biglobosa (6,48 tiges/ha), Daniellia oliveri (2,66 tiges/ha), Mangifera indica (6,48 tiges/ha), Borassus aethiopum (1,74 tiges/ha) et Tamarindus indica (1,44 tiges/ha). La surface terrière totale est de $177,353 \mathrm{~m}^{2}$ et la surface terrière moyenne est de $4,077 \mathrm{~m}^{2} / \mathrm{ha}$. L'espèce la plus dominante dans les parcs arborés du Département de la Nya est Parkia biglobosa avec une dominance relative de $34,04 \%$ et une surface terrière de $60,38 \mathrm{~m}^{2} / \mathrm{ha}$; elle est suivie de Vitellaria paradoxa qui a une dominance relative de $25,63 \%$ et une surface terrière de $45,46 \mathrm{~m}^{2} / \mathrm{ha}$ et de Daniellia oliveri avec une dominance relative $10,47 \%$ et une surface terrière de $18,58 \mathrm{~m}^{2} /$ ha (Tableau 1 ). Les autres espèces sont les moins dominantes avec moins de $1 \mathrm{~m}^{2} /$ ha de surface terrière.

\section{Abondance, dominance et indice d'importance des familles}

La famille la plus importante est celle des Fabaceae avec une VIF de $121,10 \%$ suivie des Sapotaceae (VIF $=64,14 \%)$, des Anacardiaceae $(\mathrm{VIF}=25,16 \%)$ et des Arecaceae (VIF $=18,19 \%$ ) (Tableau 2).

\section{Caractéristiques écologiques des parcs arborés}

Spectres des types biologiques

Le spectre de formes biologiques détaillées des sous-types des phanérophytes montre une prédominance des mésophanérophytes $(65,51 \%)$ et des microphanérophytes $(31,03 \%)$ (Figure 4). Les mégaphanérophytes sont très peu représentés avec $3,4 \%$ de spectres bruts.

\section{Spectres des types phytogéographiques}

Du point de vue phytogéographique, les espèces soudano-zambéziennes c'est-à-dire $\mathrm{du}$ domaine de la savane dominent avec $37,93 \%$ des espèces. Elles sont suivies des espèces pantropicales $(20,68 \%)$ et des paléotropicales $(13,73 \%)$ (Figure 5).

Tableau 1 : Espèces écologiquement importantes dans les parcs arborés du Département de Nya au Tchad.

\begin{tabular}{lllll}
\hline Espèces & $\begin{array}{l}\text { Abondance } \\
\text { relative }\end{array}$ & $\begin{array}{l}\text { Fréquence } \\
\text { relative }\end{array}$ & $\begin{array}{l}\text { Dominance } \\
\text { relative }\end{array}$ & IVI \\
\hline Vitellaria paradoxa & 35,05 & 78,74 & 25,63 & 139,4 \\
Parkia biglobosa & 22,72 & 60,34 & 34,05 & 117,1 \\
Daniellia oliveri & 9,347 & 38,51 & 10,48 & 58,33 \\
Mangifera indica & 7,575 & 21,84 & 6,864 & 36,28 \\
Tamarindus indica & 5,077 & 21,26 & 7,267 & 33,61 \\
Borassus aethiopum & 6,124 & 21,84 & 4,812 & 32,77 \\
Prosopis africana & 2,256 & 13,79 & 2,273 & 18,32 \\
Burkea africana & 1,934 & 13,22 & 1,672 & 16,82 \\
Anogeissus leiocarpus & 1,45 & 10,34 & 1,214 & 13,01 \\
Diospyros mespiliformis & 0,886 & 5,747 & 0,945 & 7,579 \\
Ziziphus mauritiana & 0,806 & 5,747 & 0,373 & 6,926 \\
Detarium microcarpum & 0,967 & 4,598 & 0,345 & 5,909 \\
Pterocarpus lucens & 0,645 & 4,023 & 0,473 & 5,14 \\
Faidherbia albida & 0,483 & 3,448 & 0,606 & 4,538 \\
Terminalia laxiflora & 0,886 & 2,874 & 0,774 & 4,534 \\
Combretum glutinosum & 0,483 & 3,448 & 0,327 & 4,259 \\
Citrus limon & 0,483 & 3,448 & 0,133 & 4,065 \\
\hline
\end{tabular}




\begin{tabular}{lllll}
\hline Hymenocardia acida & 0,483 & 3,448 & 0,099 & 4,03 \\
Annona senegalensis & 0,403 & 2,874 & 0,057 & 3,333 \\
Psidium guajava & 0,483 & 2,299 & 0,209 & 2,991 \\
Azadirachta indica & 0,322 & 1,724 & 0,235 & 2,282 \\
Ximenia americana & 0,242 & 1,724 & 0,153 & 2,119 \\
Hyphaene thebaica & 0,161 & 1,149 & 0,202 & 1,512 \\
Balanites aegyptiaca & 0,161 & 1,149 & 0,116 & 1,426 \\
Anacardium occidentale & 0,161 & 1,149 & 0,097 & 1,408 \\
Ficus capensis & 0,161 & 0,575 & 0,354 & 1,09 \\
Carica papaya & 0,081 & 0,575 & 0,167 & 0,822 \\
Sclerocarya birrea & 0,081 & 0,575 & 0,041 & 0,697 \\
Strychnos spinosa & 0,081 & 0,575 & 0,034 & 0,689 \\
\hline
\end{tabular}

IVI : Indice de Valeur d'Importance.

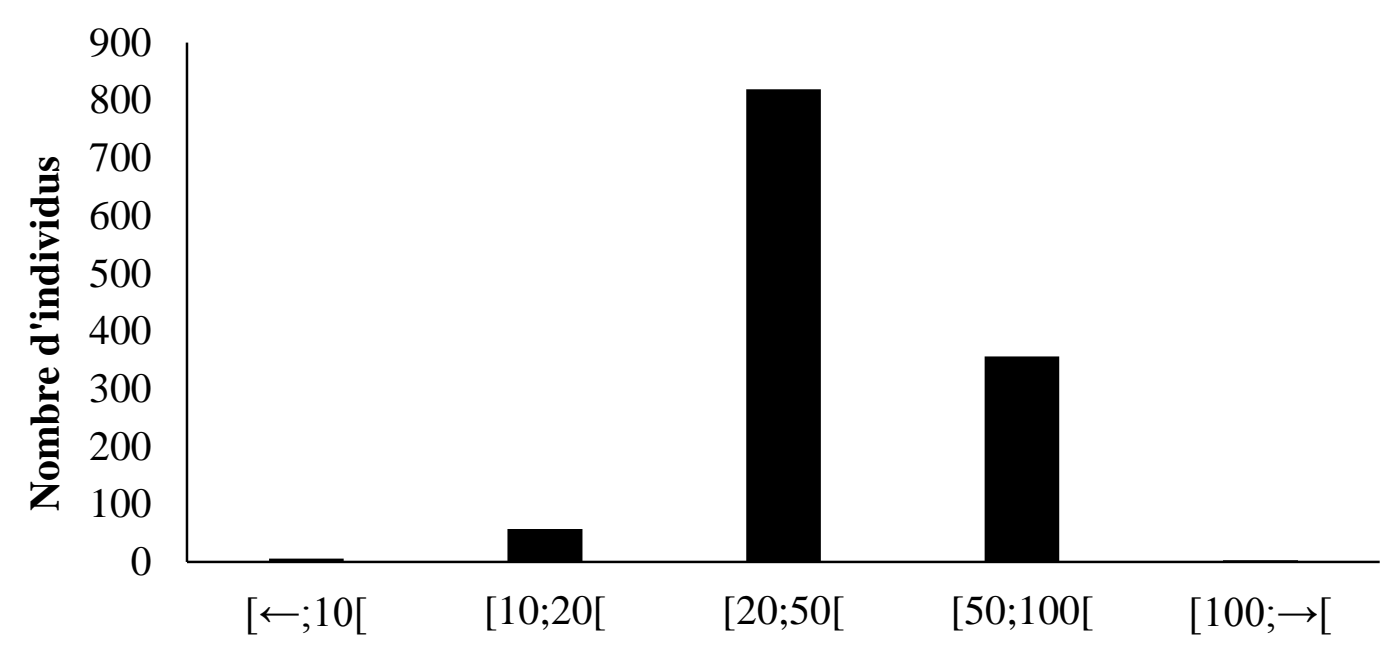

Classe de diamètre $(\mathrm{cm})$

Figure 3: Distribution des individus ligneux par classe de diamètre dans les parcs arborés du Département de Nya.

Tableau 2: Familles écologiquement importantes dans les parcs arborés du Département de Nya au Tchad.

\begin{tabular}{lcccc}
\hline Famille (APGIII) & $\begin{array}{l}\text { Diversité } \\
\text { relative }\end{array}$ & $\begin{array}{l}\text { Abondance } \\
\text { relative }\end{array}$ & $\begin{array}{l}\text { Dominance } \\
\text { relative }\end{array}$ & VIF \\
\hline Fabaceae & 24,14 & 41,5 & 55,49 & 121,12 \\
Sapotaceae & 3,448 & 35,05 & 25,63 & 64,14 \\
Anacardiaceae & 10,34 & 7,816 & 7,003 & 25,16 \\
Arecaceae & 6,897 & 6,285 & 5,013 & 18,19 \\
Combretaceae & 10,34 & 2,82 & 2,351 & 15,52 \\
Rubiaceae & 3,448 & 1,934 & 1,672 & 7,054 \\
Ebenaceae & 3,448 & 0,886 & 0,945 & 5,279 \\
Rhamnaceae & 3,448 & 0,806 & 0,373 & 4,627 \\
\hline
\end{tabular}


H. MBAIYETOM et al. / Int. J. Biol. Chem. Sci. 15(1): 68-80, 2021

\begin{tabular}{lllll}
\hline Myrtaceae & 3,448 & 0,483 & 0,209 & 4,141 \\
Rutaceae & 3,448 & 0,483 & 0,133 & 4,065 \\
Hymenocardiaceae & 3,448 & 0,483 & 0,099 & 4,030 \\
Meliaceae & 3,448 & 0,322 & 0,235 & 4,006 \\
Moraceae & 3,448 & 0,161 & 0,354 & 3,964 \\
Annonaceae & 3,448 & 0,403 & 0,057 & 3,908 \\
Olacaceae & 3,448 & 0,242 & 0,153 & 3,843 \\
Balanitaceae & 3,448 & 0,161 & 0,116 & 3,725 \\
Caricaceae & 3,448 & 0,081 & 0,167 & 3,696 \\
Loganiaceae & 3,448 & 0,081 & 0,034 & 3,563 \\
\hline
\end{tabular}

VIF : Valeur d'Importance des Familles.

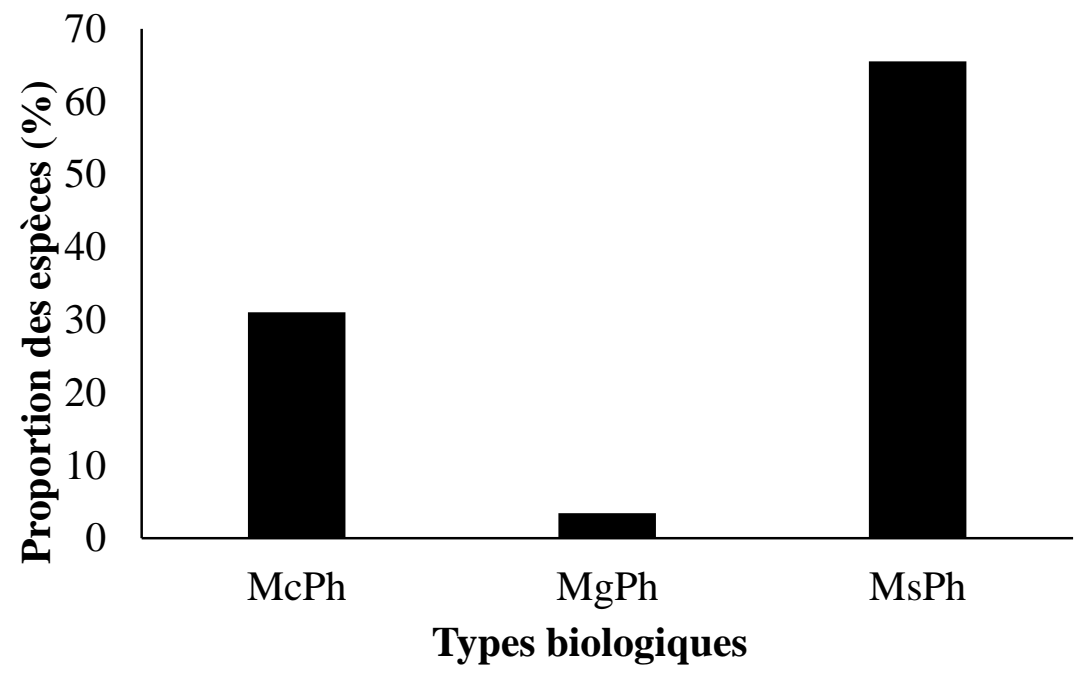

Figure 4: Spectre brut des sous-types des phanérophytes des parcs arbores du Département de Nya au Tchad. MsPh: mésophanérophytes; McPh: microphanérophytes; MgPh: mégaphanérophytes.

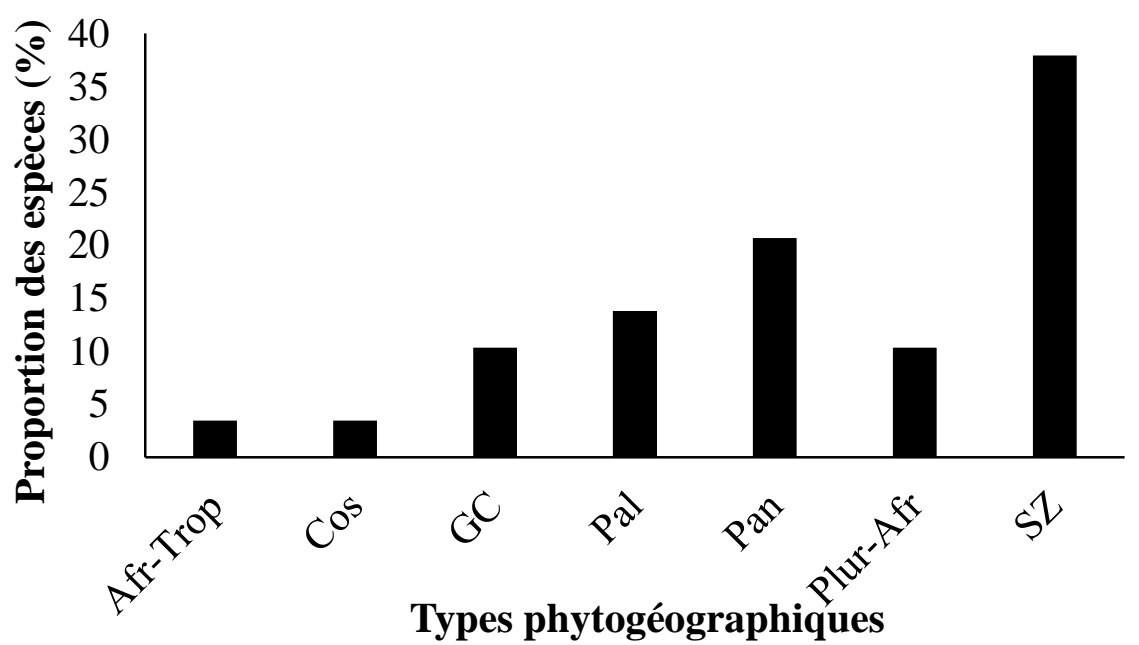

Figure 5: Spectre brut des types phytogéographiques. GC: guinéo-congolaises; Afr-Trop: afro-tropicale; Cos : cosmopolites SZ: soudano-zambéziennes; Pan: pantropicales; Paléo: paléotropicales; Plur-Afr : plurirégional africaines. 


\section{DISCUSSION}

\section{Diversité floristique des parcs arborés}

La richesse spécifique des parcs arborés du Département de la Nya est comparable à celle trouvée par Morou et al. (2016) qui ont recensés 37 espèces dans les parcs agroforestiers du terroir de Dan Saga. Ces valeurs de nombre d'espèces sont supérieures à celles trouvées dans les parcs à Neocarya macrophylla dans la zone sahélienne par Dan Guimbo et al. (2010) et dans les parcs à Balanites aegyptiaca de l'Ouest nigérien (Idrissa et al., 2018). Par contre, cette richesse spécifique est moins importante que celle obtenue par Ousmane et al. (2017) qui ont recensé 46 espèces réparties en 38 genres et 22 familles dans le terroir de Guidan Roumdji et par Amani (2016) qui a trouvé 55 espèces ligneuses réparties en 23 familles dans la zone sahélienne et nord-soudanienne au Niger.

La valeur de l'indice de diversité de Shannon indique une diversité moyenne au sein des parcs. Cette diversité moyenne observée dans ces parcs serait liée à la forte pression anthropique d'une part et aux conditions climatiques précaires d'autre part. L'équitabilité de Piélou montre qu'il existe une équirépartition des individus entre les espèces. Ces indices sont supérieurs à ceux calculés dans le parc à Guiera senegalensis et Piliostigma reticulatum situé dans la localité de Guidan Roumdji (Ousmane et al., 2017).

\section{Caractéristiques floristiques}

La faible fréquence des espèces traduit la dégradation ou sahélisation du site évoquée par certains auteurs (Garba et al., 2012; Melom et al., 2015 ). L'expression des fréquences permet aussi de montrer que certaines espèces à hautes valeurs utilitaires comme Anogeissus leiocarpa et Balanites aegyptiaca font partie des espèces peu fréquentes. Ces espèces seraient donc vulnérables aux pressions anthropiques en particulier à l'extension des surfaces cultivées, a la collecte excessive de leurs produits et à la récolte du bois pour divers usages dont principalement le chauffage (Habou et al., 2020). Elles méritent à cet effet une attention particulière dans le cadre de la gestion des ressources végétales de la zone d'étude.

La forte abondance de Vitellaria paradoxa et de Parkia biglobosa fait penser que les conditions environnementales leurs sont favorables et leur importance socioeconomique favorise leur conservation dans les parcs. En effet, Yaméogo et al. (2005) considéraient que le choix des espèces végétales à conserver, couper ou brûler obéit à certaines logiques paysannes lors du défrichement des jachères. Des espèces présentant un intérêt économique et/ou social telles que Faidherbia albida, Mangifera indica, Parkia biglobosa, Tamarindus indica et Vitellaria paradoxa sont privilégiées et conservées lors des défrichements. Les fortes valeurs d'IVI de ces espèces pourraient être liées à leur forte capacité de multiplication par voix végétative et graine (Bellefontaine et al., 2005 ; Habou et al., 2020).

Les espèces de faibles valeurs d'IVI devraient être priorisées dans les mesures de conservation pour éviter leur extinction au niveau locale (Arouna et al., 2016). Les mesures de conservation comme les plantations, la régénération naturelle assistée et la domestication peuvent être préconisées pour ces espèces. En absence de semencier ou d'attaque très probable de semences par les parasites, l'induction de la multiplication végétative peut permettre la pérennité de certaines espèces en particulier dans le milieu sahélien où le stress hydrique est très prononcé et les perturbations d'origine pastorale et anthropique sont élevées (Bellefontaine et al., 2005 ; Barmo et al., 2019).

La dominance des familles de Fabaceae et de Combretaceae dans les zones soudanosahéliennes a été déjà mise en évidence dans plusieurs travaux antérieurs (Dan Guimbo et al., 2010 ; Massaoudou et Larwanou, 2015 ; Amani, 2016; Morou et al., 2016 ; Ousmane et al., 2017; Idrissa et al., 2018). Cette dominance pourrait être due à l'adaptabilité, au mode de dissémination et à l'utilité des espèces. En effet, les Fabaceae, généralement fourragères, aux semences zoochores sont disséminées par les herbivores qui les consomment alors que les Combretaceae sont 
caractérisées par leurs fruits ailés facilement disséminés par le vent (Ouedraogo, 2009). L'importance de ces familles réside par le fait qu'elles renferment des espèces qui résistent notamment au manque et à l'insuffisance des pluies, mais aussi aux fortes températures (Savadogo et al., 2016).

\section{Structure diamétrique}

L'abondance des adultes dans ces parcs permet de dire qu'il n'y a pas un renouvellement constant des jeunes individus. La faible densité des jeunes individus ]0; 10 [ $\mathrm{cm}$ témoigne d'un environnement fortement perturbé avec semble-t-il soit une surexploitation des jeunes individus ou bien que la régénération des espèces caractéristiques du parc ne présage pas une pérennité de celuici si des mesures de régénération ne sont pas prises. Cela serait sans doute liée au manque de suivi et de protection des jeunes pousses contre les animaux et lors des défrichements. En effet, dans les parcs arborés, les arbres sont laissés par les agriculteurs soit pour leur utilité comme bois d'œuvre (Borassus aethiopium, Combretum glutinosum et Sclerocarya birrea), bois-énergie (Combretum glutinosum et Parkia biglobosa) bien reconnues par les communautes locales. A cet effet, Yaméogo (2013) a montré que les arbres des parcs arborés au Burkina Faso sont principalement utilisés pour satisfaire les besoins alimentaires et médicinaux (humains et animaux), tout en reconnaissant le faible taux de régénération des espèces retrouvées dans ces parcs. La présence des individus de grands diamètres dans les parcs pourrait indiquer qu'ils regorgent un potentiel en semenciers qui pourraient contribuer à leur stabilité et offrir une capacité de renouvellement de la ressource (Ngom et al., 2013).

\section{Densité et surface terrière des espèces dans les parcs arborés}

La faible valeur de densité moyenne des individus des parcs arborés s'expliquerait par le fait que ces espèces sont exploitées par les populations pour la satisfaction de leurs besoins quotidiens. Le même constat a été fait par plusieurs auteurs (Lawanou et al., 2005 ;
Bakhoum et al., 2012; Gonzalez et al., 2012; Ouango et al., 2015) pour qui les faibles densités sont liées à l'action anthropique (feu de brousse, coupe abusive) et aux épisodes de sécheresse et de changement climatique qu'a connu et connaît encore le Sahel. A ces facteurs, on peut aussi ajouter l'impact des surpâturages qui peut contribuer à la disparition des certaines espèces végétales dans le milieu.

Les valeurs observées pour les surfaces terrières moyenne des espèces pourraient être dues à la forte pression (coupe abusive de bois, émondage, etc) qu'exerce la population sur ces parcs. Des mesures de conservation in situ doivent être envisagées pour favoriser la production et la germination des diaspores, le recrutement des jeunes individus et la protection des adultes surtout pour les espèces des faibles valeurs d'IVI.

\section{Caractéristiques écologiques des parcs arborés \\ Spectres biologiques et phytogéographiques}

$\mathrm{La}$ forte représentation des mésophanérophytes et des microphanérophytes montre une prépondérance des formations arbustives dans les parcs arborés de la zone d'étude (Melom et al., 2015). Ces résultats confirment l'assertion de Schmidt et al. (2005) selon laquelle les types biologiques reflètent non seulement les paramètres structuraux dans une végétation mais également les conditions environnementales variées.

$$
\text { L'analyse des spectres }
$$
phytogéographiques met en évidence la dominance des espèces soudano-zambéziennes dans les parcs, attestant que la flore de la zone soudanienne garde encore sa spécificité phytogéographique en dépit des perturbations enregistrées. Cette forte proportion des espèces soudano-zambéziennes traduit l'appartenance de la zone d'étude au domaine soudanien. L'abondance des espèces à large distribution (pantropicales et paléotropicales) et à distribution continentale (afro-tropicales) indique que la zone du Département de la Nya appartiendrait au domaine soudanien perturbé (Sinsin, 2001). Ces résultats sont comparables 
à ceux de Mahamane et al. (2007) qui révèlent la dominance de ces types phytogéographiques dans tout l'Ouest du Niger. Ce constat a été également fait par Barmo et al. (2019) dans des parcs agroforestiers adjacents à la forêt protégée de Baban Rafi au Niger et par Arouna et al. (2016) dans la zone soudanienne du Benin.

\section{Conclusion}

Cette étude a permis une meilleure connaissance des caractéristiques floristiques, structurales et écologiques de la végétation ligneuse dans les parcs arborés du Département de la Nya. La végétation ligneuse de la zone est assez pauvre et est composée de 29 espèces reparties en 29 genres et 18 familles. Les indices de diversité de Shannon et d'équitabilité de Piélou révèlent qu'il y a une diversité moyenne. Les espèces écologiquement importantes sont Vitellaria paradoxa, Parkia biglobosa et Daniellia oliveri. La famille des Fabaceae est la plus riche en espèces suivie des Anacardiaceae et des Combretaceae. Les familles les plus importante sont les Fabaceae, les Sapotaceae, les Anacardiaceae et les Arecaceae. Les types biologiques dominants sont les mésophanérophytes alors que les types phytogéographiques les plus représentés sont les espèces soudano-zambéziennes mais avec la présence des espèces pantropicales. Les résultats de ces travaux permettent de conclure que la flore ligneuse des parcs arborés soudaniens au Tchad s'appauvrirait au dépend d'une poignée d'espèces utiles préservées par les populations pour leurs produits et services. Cette modification structurelle et fonctionnelle mériterait d'être mieux caractérisée afin d'en identifier les facteurs déterminants et les stratégies pour une gestion intégrée et durable.

\section{CONFLIT D'INTERETS}

Les auteurs de ce manuscrit déclarent qu'ils n'ont aucun conflit d'intérêts.

\section{CONTRIBUTIONS DES AUTEURS}

MH a collecté les données, MH et WTJB ont analysées les données et écrire le manuscrit, ATML et TNM ont supervisé tout le travail, tous les auteurs ont lu et approuvé le manuscrit final.

\section{REMERCIEMENTS}

Les auteurs remercient les chefs de Cantons et les populations du Département de la Nya pour la disponibilité des données et d'échanges fructueux.

\section{REFERENCES}

Akinnifesi FK, Sileshi G, Ajayi OC, Chirwa PW, Kwesiga F, Harawa R. 2008. Contributions of agroforestry research and development to livelihood of smallholder farmers in Southern Africa: 2. Fruit, medicinal, fuelwood and fodder tree systems. Agricultural Journal., 3(1): 76-88.

Amani A. 2016. Croissance et potentiel de séquestration de carbone de quatre espèces de Combretaceae en zone sahélienne et nord-soudanienne au Niger (Afrique de l'Ouest). Thèse de Doctorat en biologie et écologie végétales, Université Abdou Moumouni de Niamey, Niger, $184 \mathrm{p}$.

Arbonnier M. 2002. Arbres, arbustes et lianes d'Afrique de l'Ouest ( $\left.2^{\mathrm{eme}} \mathrm{edn}\right)$. CIRADMNHN- UICN ; 541 p.

Arouna O, Etene CG, Issiako D. 2016. Dynamique de l'occupation des terres et état de la flore et de la végétation dans le bassin supérieur de l'Alibori au Benin. $J$. Appl Biosc., 108: 10531 - 10542. http://dx.doi.org/10.4314/jas.v108i1.7

Bakoum C, Agbangba EC, Ndour B. 2012. Natural regeneration of tree in arid and semi-arid zones in West Africa. Journal of Asian Scientific Research., 2(12) 820834.

Barmo S, Amani A, Soumana I, Ichaou A, Karim S, Mahamane A. 2019. Structure et diversité des parcs agroforestiers adjacents à la forêt protégée de Baban Rafi, Niger - Afrique de l'Ouest. Afrique Sciences., 15(2) : 166 - 185.

Bellefontaine R, Sabir M, Kokou K, Guinko S, Saadou M, Ichaou A, Hatem C, Bationo BA, Karim S, Dourma M. 2005. Argumentaire pour l'étude et l'utilisation 
des marcottes et drageons dans les pays à faible couvert ligneux. Sècheresse, 16(4): 312-314.

Dan Guimbo I, Mahamane A, Ambouta JMK. 2010. Peuplement des parcs à Neocarya macrophylla (Sabine) Prance et à Vitellaria paradoxa (Gaertn. CF) dans le sud-ouest nigérien : diversité, structure et régénération. Int. J. Biol. Chem. Sci., 4(5) $1706-1720$.

Garba I, Touré I, Ickowicz A, Césaro JD. 2012. Evolution historique de la pluviosité. In: Système d'information sur le pastoralisme au Sahel. Atlas des évolutions des systèmes pastoraux au Sahel 1970-2012. FAO et CIRAD (eds), pp. 8-11.

Gbesso F, Yedomonhan H, Tenté B, Akoegninou A. 2014. Distribution géographique des populations de rôniers (Borassus aethiopum Mart, Arecaceae) et caractérisation phytoécologique de leurs habitats dans la zone soudano-guinéenne du Benin. J. Appl. Biosci., 74 : $6099-$ 6111.

DOI: https://doi.org/10.4314/jab.v74i1.14

Glele kakai R, Salako VK, Lykke AM 2016. Techniques d'échantillonnage en étude de la végétation. Annales des Sciences Agronomiques, 20(spécial projet Undesert-UE): 1-13.

Gonzalez P, Tucker CJ, Sy H. 2012. Tree density and species decline in the African Sahel attributable to climate. J. Arid. Environ., 78: 55-64. DOI : https://doi.org/10.1016/j.jaridenv.2011.1 1.001

Habou R, Massaoudou M, Abasse T, Mahamane A, Larwanou M, Van Damme $P$ 2020. Structure et régénération des peuplements naturels de Balanites aegyptiaca (L)DEL et Ziziphus mauritiana Lam suivant un gradient écologique dans la région de Maradi au Niger. Afrika Focus 33(1): 83-104.

Idrissa B, Soumana I, Issiaka Y, Ambouta JMK, Mahamane A, Saadou M, John CW. 2018. Trend and Structure of Populations of Balanites aegyptiaca in Parkland Agroforests in Western Niger.
Annual Research \& Review in Biology., 22(4): $\quad 1-12 . \quad$ DOI: https://doi.org/10.9734:ARRB/2018/386 50

Kent M, Coker P. 2003. Vegetation description and Analysis. A Pratical Approch. John Willey et Sons Eds, Belhaven Press: London; $363 \mathrm{p}$.

Larwanou M, Saadou M. 2005. Biodiversity of ligneous species in semi-arid to arid zones of southwestern Niger according to anthropogenic and natural factors. Agri. Ecosys. Environ., 105: 267-271. DOI: https://doi.org/10.1016/j.agee.2004.03.00 4

Mahamane A, Ichaou A, Ambouta JMK, Saadou M, Morou B, Amani I, Hango M, Herbes JMD, Gineste P, Wata I, Abassa I. 2007. Indicateurs écologiques de la période optimale de remise en culture de jachères au Niger. Sécheresse., 18(4) : $289-295$.

Manzo OL, Garba OB, Morou B, Karim S, Mahamane A. 2017. État de la végétation ligneuse au Sahel : Cas de Guidan Roumdji au sahel central du Niger. $J$. Anim. Plant. Sci., 31(3): 5033-5049.

Massaoudou M, Larwanou M. 2015. Caractérisation des peuplements ligneux des parcs à Faidherbia albida (Del) A. Chev. et à Prosopis africana (Guill., Perrot et Rich.) Taub du Centre-Sud Nigérien. J. Appl. Biosci., 94 : 8890 8906.

DOI : https://doi.org/10.4314/jab.v94i1.6

Mbaygone E. 2008. Flore et végétation de la réserve partielle de faune de Pama, sudest du Burkina Faso. Thèse de doctorat, Université de Ouagadougou, p. 138.

Melom S, Mbaygone E, Bechir AB, Ratnan N, Mapongmetsem PM. 2015. Caractéristiques floristique et écologique des formations végétales de Massenya au Tchad (Afrique centrale). J. Anim. Plant. Sci., 25(1): 3799-3813.

Morou B, Ounani H, Abdoulaye AO, Diouf A, Guero C, Mahamane A. 2016. Caractérisation de la structure démographique des ligneux dans les parcs agroforestiers du terroir de Dan Saga 
(Aguié, Niger). Int. J. Biol. Chem. Sci., 10(3): 1295-1311. DOI: https://doi.org/10.4314/ijbcs.v10i3.31

Ngom D, Fall T, Sarr O, Diatta S, Akpo LE. 2013. Caractéristiques écologiques du peuplement ligneux de la réserve de biosphère du Ferlo (Nord Sénégal). $J$. Appl. Biosci., 65: 5008-5023. DOI : https://doi.org/10.4314/jab.v65i0.89644

Ouango MS, Korodjouma O, Jennie B, Issa O, Line G, Elin E, Nabsanna PZ. 2015. Etats des écosystèmes sahéliens: reverdissement, perte de la diversité et qualité des sols. Afrique Sciences., 11(5): $433-446$.

Ouedraogo O. 2009. Phytosociologie, dynamique et productivité de la végétation du parc national d'Arly (SudEst du Burkina Faso). Thèse de doctorat de l'Université de Ouagadougou, p. 188

Oumorou M, Sinadouwirou T, Kiki M, Glélé R, Mensah GA, Sinsin B. 2010. Disturbance and population structure of Vitex doniana $\mathrm{Sw}$; in northern Benin, West Africa. Int. J. Biol. Chem. Sci., 4(3): 624-632.

DOI: https://doi.org/10.4314/ijbcs.v4i3.60467

Ousmane LM, Oumarou BG, Morou B, Karim S, Mahamane A. 2017, État de la végétation ligneuse au Sahel : Cas de Guidan Roumdji au sahel central du Niger. J. Anim. Plant. Sci., 31(3) : 5033 5049.

Savadogo OM, Ouattara K, Pare S, Ouedraogo I, Sawadogo-Kaboré S, Barron J, Zombre NP. 2016. Structure, composition spécifique et diversité des ligneux dans deux zones contrastées en zone
Sahélienne du Burkina Faso. VertigO., 16(1), mis en ligne le 09 mai 2016, consulté le 08 octobre 2019. DOI: https://doi.org/10.4000/vertigo.17282

Schmidt M, Kreft H, Thiombiano A, Zizka G. 2005. Herbarium collection and field data-based plant diversity maps for Burkina Faso. Diversity and Distribution, 11: 509-516. DOI : https://doi.org/10.1111/j.13669516.2005.00185.x

Sinsin B. 2001. Formes de vie et diversité spécifique des associations de forêts claires du nord du Bénin. Systematic Geographic of Plant, 71 : 873-888. DOI: https://doi.org/10.2307/3668725

Thiombiano A, Glele kakai R, Bayen $\mathrm{P}$, Boussim JL, Mahamane A. 2016. Méthodes et dispositifs d'inventaires forestiers en Afrique de l'ouest : état des lieux et propositions pour une harmonisation in Méthodes de collecte et d'analyse des données de terrains pour l'évaluation et le suivi de la végétation en Afrique. Annales des Sciences Agronomiques., $\mathbf{2 0}$ (Spécial projet Undesert-UE): 15-30.

Wouokoue TJB. 2018. Phytosociologie des monts Bambouto et Mbapit, équations allométriques pour l'estimation de la biomasse aérienne des ligneux des savanes. Thèse de doctorat/Ph.D, Université de Dschang, p. 228.

Yaméogo JT, Somé AN, Hien M. 2009. Étude préliminaire à une restauration de sols dégradés en zone soudanienne du Burkina Faso. Sécheresse, 20(1): 8-32. DOI : https://doi.org/10.1684/sec.2009.0216. 